\title{
Traduire
}

Une autre perspective sur r tr traduction

Revue française de la traduction

$235 \mid 2016$

Luxe, mode... et traduction !

\section{Emprunts et malentendus dans la mode italienne}

\section{Anna Rita Parente}

\section{OpenEdition}

Journals

Édition électronique

URL : https://journals.openedition.org/traduire/850

DOI : $10.4000 /$ traduire.850

ISSN : 2272-9992

Éditeur

Société française des traducteurs

Édition imprimée

Date de publication : 1 décembre 2016

Pagination : 66-68

ISSN : 0395-773X

\section{Référence électronique}

Anna Rita Parente, «Emprunts et malentendus dans la mode italienne », Traduire [En ligne], 235 | 2016,

mis en ligne le 01 décembre 2018, consulté le 06 juillet 2021. URL : http://journals.openedition.org/ traduire/850 ; DOI : https://doi.org/10.4000/traduire.850 


\section{Emprunts et malentendus dans la mode italienne}

\section{Anna Rita Parente}

L'ouverture de la langue italienne aux emprunts lexicaux est un phénomène connu de longue date qui s'est développé assez naturellement. Le français en particulier a représenté pendant plusieurs siècles la langue de la Culture avec un grand C. Aux xvie et XVIIe siècles, dans les domaines du costume, de la gastronomie et des arts décoratifs, les références au français(1) sont particulièrement fréquentes. Avec le temps, cette tendance s'est généralisée, en s'étendant à d'autres langues, dont l'anglais, et à d'autres secteurs. La politique d'autarcie linguistique voulue par le régime fasciste n'a guère eu d'effet et, en dépit des interdictions et des mesures coercitives imposées durant cette période, on a recommencé dès la fin de la Seconde Guerre mondiale à puiser abondamment dans les nouvelles langues dominantes, l'anglais américain en tête(2).

L'italien de la mode ne fait pas exception ; il regorge même d'emprunts lexicaux à d'autres langues parmi lesquelles le français fait incontestablement figure de langue de référence. Ces emprunts sont parfois injustifiés, soit parce qu'il existe un terme équivalent en italien, soit parce qu'ils sont utilisés de manière impropre, dénaturant le sens original du terme français. C'est le cas particulièrement emblématique du mot chiffon, très largement utilisé dans le monde de la mode italienne pour désigner un type de tissu, généralement en soie, extrêmement léger et transparent. Ici, le malentendu est total et d'une certaine façon, le terme français est " ennobli » dans son acception italienne puisque l'usage qui en est fait ne renvoie en rien à son véritable sens de chiffon, guenille. En français, on traduira donc le chiffon italien par mousseline.

Dans d'autres cas, le recours aux termes français découle d'une intention délibérée de se distinguer. II implique dès lors la reconnaissance de la primauté française dans le domaine de

(1) Morgana 2010, p. 72-74.

(2) Morgana 2010, p. 106-109. 
la mode. Des termes comme prêt-à-porter ou haute couture sont entrés tels quels dans le vocabulaire italien. Le fait est que Paris conserve - aujourd'hui encore - l'exclusivité des défilés de haute couture, héritage d'une ancienne tradition d'artisanat d'art remontant à Charles Frederick Worth, fondateur en 1858 du premier atelier de haute couture dans la capitale française(3). Quelques Maisons italiennes ont sollicité et obtenu de la Chambre syndicale de la Couture parisienne le droit d'accéder à ce club restreint. Ainsi, à l'occasion des défilés de haute couture qui se déroulent à Paris deux fois par an, de précieuses pièces de vêtements extrêmement coûteuses sont transférées à Paris, sans obligation d'ailleurs pour les créateurs concernés de renoncer aux passerelles milanaises du prêt-à-porter, tout aussi prestigieuses. Si Paris reste la reine de la mode, le français en reste la langue. Qu'il s'agisse de la description des looks de défilé ou des articles des principales revues spécialisées de mode, l'italien recourt fréquemment aux gallicismes. Cette tendance doit être interprétée non seulement comme un hommage et une forme de reconnaissance de la primauté française, mais également comme une volonté de représenter le luxe et l'élégance du produit.

Rien d'étonnant dès lors que l'on préfère l'emploi de termes français tels que satin, crêpe, revers, papillon ou bustier aux moins glamour raso, crespo, bavero, farfallino, corpino. De la même manière, dans la description des tissus de la haute couture, on recourt largement aux gallicismes comme cannelé pour cannettato, plissé pour plissettato, froissé pour stropicciato, lamé pour laminato, à carreaux plutôt qu'a quadri et à damier plutôt qu'a scacchi pour ne citer que quelques exemples.

Plus récent, l'emploi de termes d'anglais américain que l'on retrouve d'ailleurs également dans la langue française témoigne d'une réalité mondialisée dans laquelle l'anglais s'est affirmé comme la langue commune de référence. C'est ainsi que des mots italiens comme pantaloncini, maglietta, maglione, impermeabile ont été bannis au profit des shorts, pullover et autre trench, ce dernier s'étant popularisé en Italie sous l'influence d'Humphrey Bogart dans le film Casablanca (1942).

Parfois, l'équivalent français a été remplacé après plusieurs années de bons et loyaux services par le terme anglais, jugé plus contemporain. Tel est le cas par exemple d'un article très en vogue dans les années 1980, le fuseau (transcrit littéralement en italien par fusò ou plus rarement désigné par deux néologismes créatifs : pantacalza ou pantacollant). Ce pantalon moulant en tissu extensible récemment revenu à la mode est désormais appelé legging. La préférence pour les termes d'anglais américain au détriment des français est souvent interprétée en termes de modernité. II en est ainsi du mot make-up, qui a maintenant supplanté en italien le " vieux " maquillage, comme du beauty case qui a remplacé le nécessaire.

(3) Worsley 2011, p. 8. 
Même dans le cas d'un accessoire aussi emblématique de l'excellence italienne que les chaussures, l'influence de mots étrangers est évidente. Le passage des scarpe da ginnastica du vestiaire sportif à celui du quotidien s'est accompagné d'un changement de dénomination et de l'affirmation du terme sneakers. C'est au français en revanche qu'est emprunté le terme plateau qui a totalement supplanté l'italianissime zeppa, pour désigner ce talon qui s'affine progressivement en triangle, une invention pourtant purement italienne(4).

Comme l'illustrent ces exemples, l'emprunt lexical dans l'univers de la mode ne vise pas, dans la plupart des cas, à combler des lacunes de l'italien. II répond davantage à une volonté de s'adapter aux tendances du moment. L'italien de la mode adopte tantôt un terme étranger tantôt un autre avec une certaine désinvolture : peu importe son origine dès lors qu'il permet de donner du relief à l'écrit et de le rendre plus " chic »... en français dans le texte évidemment !

annaritaparente@gmail.com

Docteur en histoire ancienne, Anna Rita Parente a travaillé longtemps dans l'enseignement universitaire et dans la recherche en histoire et archéologie. Installée en France depuis 2009, elle a créé sa société en 2013 : Zetema, un mot d'origine grecque qui signifie recherche, spécialisée dans la traduction français/italien, la formation et la rédaction en italien. Ses domaines d'intérêt et d'intervention sont prioritairement le luxe, l'art et l'histoire, ainsi que le tourisme et les loisirs.

\section{Bibliographie}

DONNANNO Antonio, 2011, Modabolario. Parole e immagini della moda. Dizionario tecnicocreativo, Milan (Italie), Ikon.

MORGANA Silvia, 2010, Breve storia della lingua italiana, Rome, Carocci.

VERGANI Guido, 2009, Dizionario della moda, Milan (Italie), Baldini Castoldi Dalais Editore.

WORSLEY Harriet, 2011, Cento idee che hanno vestito la moda, Modène (Italie), Logos.

(4) Worsley 2011, p. 90. 